\title{
Impact of an integrated core/SOL description on the optimization of tokamak fusion reactors
}

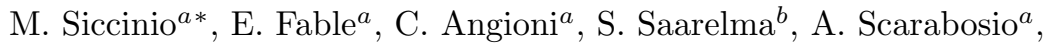 \\ H. Zohm ${ }^{a}$ \\ ${ }^{a}$ Max Planck Institut für Plasmaphysik, Boltzmannstr. 2, \\ 85748 Garching bei München, Germany \\ ${ }^{b}$ Culham Centre for Fusion Energy, Culham Science Centre, \\ Abingdon, United Kingdom
}

\begin{abstract}
An updated and improved version of the OD divertor and scrapeoff layer (hencefort SOL) model published in [1] has been coupled with the $1.5 \mathrm{D}$ transport code ASTRA $[2,3]$. The resulting numerical tool has been employed for various scans in the major radius $R$ and in the toroidal magnetic field $B_{T}$ - for different aspect ratios $A$, safety factors $q$, allowable loop voltages $V_{\text {loop }}$ and $\mathrm{H}$ factors - in order to identify the most convenient choices for an electricity producing tokamak. Such scenario analysis has been carried out evaluating self-consistently, and simultaneously, both the core profile and transport effects, which significantly impact on the fusion power outcome, and the divertor heat loads, which represent one of the most critical issues in view of the realization of fusion power plants $[4,5]$. The main result is that, when divertor limits are enforced, the curves at constant electrical power output are closed on themselves in the $R-B_{T}$ plane, and a maximum achievable power exists - i.e. no benefits would descend from a further increase of $R$ and $B_{T}$ once the optimum has been reached. This result appears as an intrinsic physical limit for all those devices where a radiative SOL is needed to deal with the power exhaust, and where a lower limit on the power crossing the separatrix (e.g. because of the L-H transition) is present.
\end{abstract}

\section{Introduction}

In the course of the currently ongoing pre-conceptual design analysis phase for the prototypical fusion reactor DEMO [6], the necessity of a comprehensive approach to the machine design in order to guarantee the simultaneous fulfilling of all physical and technological constraints has become apparent. For this reason, many so called system codes, like for example PROCESS [7, 8] have been and still are being developed, continuously improving their accuracy. Most system codes consist of various interdependent sub-units, each of which referring to a particular aspect of the machine physics or technology. Every set of engineering parameters (e.g. major radius $R$, toroidal field $B_{T}$, aspect ratio $A$ and so on) which allows all constraints in the various sub-modules to be satisfied at the same time represents, in principle, a feasible fusion reactor with a corresponding operational point. As the number of sub-units is most of the

*Contact: mattia.siccinio@ipp.mpg.de 
times pretty large, the physical models which are employed have to be quite simple, in order to mantain the computational time at an acceptable level. Thus, for example, the density and temperature profiles, which determine the achievable fusion power, playing therefore a central role in the design of fusion power plants, are just assumed to possess some given shape only partially adjustable. In the present work, the 1.5 transport code ASTRA $[2,3]$ has been coupled to an ameliorated version of the OD SOL and divertor model published in [1] with the aim of investigating the performance of different machines, and scenarios, with a much higher degree of detail and physical plausibility than every nowadays existing system code, keeping nevertheless the computational times low. In particular, we have carried out various (geometrical) major radius $R$ and toroidal magnetic field $B_{T}$ scans - for different values of the aspect ratio $A$, of the allowable loop voltage $V_{\text {loop }}$ and $\mathrm{H}$ factor (which is introduced as the ratio between the global energy confinement time $\tau_{E}$ and the value of $\tau_{E}$ obtained via the well-known IPB98(y,2) scaling $\left.[9,10]\right)$ - with the precise goal of identifying the most appropriate choices, from the standpoint of plasma physics, for the production of electrical power.

The inclusion of a SOL and divertor model is of primary importance for such investigation, as it is well-known $[4,5]$ that one of the major problems for most reactor scenarios (see e.g. $[11,12]$ ) is to keep the heat flux on the divertor plates below the technological limit of $10 \mathrm{MW} / \mathrm{m}^{2}[13,14]$. This requirement can only be fulfilled with a strong degree of plasma detachment from divertor targets, as in view of the widely employed Eich scaling for attached divertor conditions $[15,16]$, the surface on which the power exhaust is deposited would otherwise be too small, leading to unacceptably high power densities. Thus, an evaluation of the degree of divertor detachment is mandatory to correctly identify the feasible operating points for fusion machines. Here, it is shown that the radiating impurities, which have to be present in the SOL and in the core with a sufficiently high concentration to protect the divertor, can heavily deteriorate the fusion power outcome via fuel dilution.

The main result we present in this work is that the curves at constant electrical power output are closed on themselves when plotted on a $R-B_{T}$ plane, which means that it is not in general true that an increase in $R$, or $B_{T}$, or even both necessarily leads to an improvement in the machine performance. The present analysis mainly focuses on the plasma physics aspects of the problem, considering only the striking power on the divertor plates as technological constraint. In section 3.4, however, a brief discussion about further technological aspects is given. Also, we will discuss one recently proposed reactor design [17] in view of our results.

This paper is structured as follows: in section 2, the employed ASTRA settings, the chosen configuration requirements and the coupling with the $0 \mathrm{D}$ SOL model are described. In section 3 , the results of the performed scans are 
shown and extensively discussed. Conclusions are drawn in section 4, whereas appendix I and II contain the details about the ASTRA transport model and the 0D SOL model, respectively.

\section{The model}

\subsection{ASTRA settings}

The $1.5 \mathrm{D}$ transport code ASTRA $[2,3]$ is a widely employed numerical tool for the investigation of particle and heat transport in tokamaks. The code is characterised by a remarkable flexibility on the transport models which can be utilised, both for energy and for particles. To improve the readability of the present manuscript, all the details about the transport model which has been chosen for the present investigation are reported in appendix I. Here, we limit ourselves to mention the features which are necessary for the comprehension of the results in the following.

The power balance is performed separately for ions and electrons. The total power heating the electron species $P_{e}$ is defined as

$$
P_{e}=P_{\alpha, e}+P_{a u x, e}-P_{r a d}-P_{e, i},
$$

where $P_{\alpha, e}$ is the fraction of the fusion born $\alpha$-particle power $P_{\alpha}$ absorbed by electrons, $P_{a u x, e}$ is the fraction of auxiliary heating power $P_{a u x}$ absorbed by electrons, $P_{\text {rad }}$ represents the radiative losses (including line radiation from impurities, bremsstrahlung and synchrotron radiation) and $P_{e, i}$ is the classical equipartition power from electrons to ions - which clearly can become negative where ions are hotter than electrons. The corresponding $P_{i}$ for the ions reads instead

$$
P_{i}=P_{\alpha, i}+P_{a u x, i}+P_{e, i},
$$

where $P_{\alpha, i}$ is the fraction of $P_{\alpha}$ absorbed by ions and $P_{a u x, i}$ is the fraction of $P_{\text {aux }}$ absorbed by ions. The total fusion power $P_{\text {fus }}$ (which is connected to $P_{\alpha}$ through the well-known relation $\left.P_{\alpha}=1 / 5 P_{\text {fus }}\right)$ is calculated self-consistently employing the ion density and temperature profiles, accounting for the dependence of the fusion cross section on the ion temperature - in particular, the "less-than-quadratic" behaviour for $T_{i}$ much larger than $20 \mathrm{keV}$, which leads to a saturation of the global fusion power outcome for increasing central temperatures, has been considered. Its subdivision into $P_{\alpha, e}$ and $P_{\alpha, i}$ is determined in ASTRA taking into account the critical energy and the collisionality, whereas $P_{a u x}$ is on the contrary assumed to be split as

$$
\begin{aligned}
& P_{a u x, e}=0.4 P_{a u x} \\
& P_{a u x, i}=0.6 P_{a u x},
\end{aligned}
$$

as more detailed determinations of such subdivision can be shown to have a limited impact on the problem. For the synchrotron radiation power $P_{\text {sync }}$, 
the formula contained in [18], which is particularly accurate at high electron temperatures, has been chosen,

$$
P_{\text {sync }} \propto\left(B T_{e}\right)^{2.5} \sqrt{n},
$$

where $T_{e}, n$ and $B$ have to be understood as local values for the electron temperature and density and for the total magnetic field, respectively. The particle transport is constrained by fixing the density at the separatrix $n_{u p}$ and the density at the pedestal top $n_{P T}$ at $0.5 n_{G W}$ and $0.9 n_{G W}$ respectively, with $n_{G W}$ indicating the Greenwald density. For this choice, which is a bit optimistic, we have assumed an improvement in the particle confinement capability of future devices in comparison to what nowadays observed in the experiments. A sensitivity study on the influence of these values on the fusion outcome is of course relevant, but goes beyond the scope of the present work.

In this analysis we consider only three impurity species, namely Ar, Xe and He. Helium is originated by the nuclear fusion reactions, and therefore its concentration is the result of the combined effect of the total amount of D-T reactions taking place and of the divertor pumping capability, the latter assumed for simplicity to be proportional to the tokamak volume - i.e. to $R a^{2}$, where $a=R / A$ is the tokamak minor radius. Again, this is an optimistic assumption, as a weaker dependence of the pumping capacity on the reactor size can be shown to lead to potentially detrimental He accumulation in the core. A more exact determination of the He pumping capability goes at present beyond the purposes of our investigation. Viceversa, argon and xenon are the puffed radiating species, the first one being mainly employed as a core radiator and the second one as a SOL and divertor radiator. Their concentration is supposed to be completely determined in magnitude by the control scheme described in subsection 2.3 , and assumed to be spatially constant throughout the core domain - this assumption applying to $\mathrm{He}$ as well. Also, no intrinsic impurity originating by the erosion of the plasma facing components is included in the present analysis, this assumption being somehow justified by the constraint of low temperature at the divertor plate, see below. On the contrary, the fuel dilution is taken into account in the ASTRA calculations when determining the fusion power.

\subsection{Coupling to the 0D divertor model}

In this subsection, the interplay between the $0 \mathrm{D}$ model and the $1.5 \mathrm{D}$ transport code ASTRA $[2,3]$ is explained. To improve the readability of the manuscript, the details about the $0 \mathrm{D}$ divertor model have been included in appendix II. The model is, as mentioned, a 0D model with loss factors, which as discussed in the appendix are calculated through a fit built on a database of SOLPS simulations with conventional divertor with vertical target and closed geometry. It receives as input at every time step the upstream density $n_{u p}$, the upstream parallel heat flux $q_{\|, u p}$ and the argon relative concentrations $c_{z, A r}^{S O L}$ and $c_{z, A r}^{d i v}$, defined respectively in the SOL and in the divertor, according to the control strategy 
discussed below. The required geometrical data of the divertor - see appendix II - are instead fixed at the beginning of each calculation and not updated during the time evolution of the profiles. The upstream parallel heat flux is calculated via

$$
q_{\|, u p}=\frac{0.5 P_{s e p}}{2 \pi R \lambda_{q}\left(B_{p} / B_{T}\right)_{O M}},
$$

where $P_{\text {sep }}$ is the plasma power crossing the separatrix, $\left(B_{p} / B_{T}\right)_{O M}$ is the ratio between the poloidal and the toroidal component of the magnetic field at the separatrix on the outer midplane (which is taken as upstream position), the factor 0.5 is included assuming a perfect subdivision of the heat exhaust between the two divertor plates and $\lambda_{q}$ represents the power decay length at the outer midplane. This quantity is calculated employing the well-known Eich scaling $[15,16]$, namely

$$
\lambda_{q} \propto B_{T}^{-0.78} q_{c y l}^{1.02} P_{\text {sep }}^{0.1} R^{0.02},
$$

where $\lambda_{q}$ is given in $\mathrm{mm}, P_{\text {sep }}$ in $\mathrm{MW}, B_{T}$ in Tesla and $q_{c y l}$ is the cylindrical safety factor. A calibration coefficient has been added to the original expression to obtain $\lambda_{q} \sim 3 \mathrm{~mm}$ for "DEMO-like" parameters (see Fig.1 below). As one can see, this scaling is very unfavourable, as it leads to higher $q_{\|, u p}$ for higher $B_{T}$, whereas no benefits come from the increase of the machine size. For simplicity, we assume the Eich scaling to be valid to describe the upstream conditions even when the divertor is detached, which is a reasonable assumption for plasmas with no deteriorated confinement.

The impurity concentrations $c_{z, A r}^{S O L}$ and $c_{z, A r}^{d i v}$ can in principle be treated as independent one on another in our model, see appendix II. In this work, however, it is assumed that their ratio remains constant throughout the calculation, i.e. that a precise compression factor between SOL and divertor exists. Also, the compression factor between the plasma core and the SOL is assumed to be constant. The toroidal flux expansion $f_{B}$ has been set to 1 (an ITER-like lower single null configuration has been assumed, thus no substantial variation of the field strength between outer midplane and plates is foreseen), whereas the geometrical factor $\eta_{B}$, linked to the angle between the magnetic field line and the divertor plate at the strike point, has been set to 10 according to the standard assumptions for DEMO (a further increase of such value is limited by engineering considerations, see [13] and references therein). Finally, the connection lengths $L_{\|}$and $L_{\text {div }}$ (identifying the distances along the open magnetic field line between the divertor plate and, respectively, the outer midplane and the divertor entrance) are calculated as

$$
\begin{array}{r}
L_{\|}=\pi R q_{c y l} \\
L_{d i v}=\frac{\pi}{3} R q_{c y l} .
\end{array}
$$

The $0 \mathrm{D}$ model provides in output the temperature, the particle flux and the heat flux at the plate. When these quantities exceed some user defined threshold value, ASTRA reacts back by increasing the argon concentration until the 
divertor is sufficiently protected. In view of the constant compression factors, this has repercussions on the impurity concentration in the core as well, negatively affecting the fusion power output via fuel dilution. The control strategy for the concentration values which has been implemented in ASTRA for the present calculations is discussed in more detail in the next subsection.

\subsection{Scenario requirements}

The scenarios considered in the present investigation have been calculated by imposing the following constraints:

- The charged particles power at the separatrix $P_{\text {sep }}$ must be comprised between $1.1 P_{L H}$ and $1.2 P_{L H}$, where $P_{L H}$ is the $L H$ transition power calculated by means of the widely employed Martin scaling [19],

$$
P_{L H}=0.049 B_{T}^{0.80} n^{0.72} S^{0.94},
$$

where $S$ is the plasma surface and $n$ is expressed in units of $10^{20} \mathrm{~m}^{-3}$.

- The temperature at the divertor must be lower than $5 \mathrm{eV}$, as this condition is typically used as a proxy for divertor detachment [5]. In reality, this condition alone is not always sufficient to guarantee under every circumstance that the heat flux on the target remains below the technological limits, nor that the level of sputtering remains low enough. However, more refined criteria would not affect the main conclusions of our work, at least from a qualitative point of view.

The first requirement is satisfied either via the control of the Xe concentration in the plasma core or by auxiliary power injection. More in detail, when $P_{\text {sep }}>1.2 P_{L H}$, ASTRA reacts back by increasing the xenon concentration. Viceversa, if $P_{\text {sep }}$ drops below $1.1 P_{L H}$, the Xe concentration is reduced, and if the condition is not fulfilled even at zero Xe concentration, the auxiliary heating system is turned on, providing the required power $P_{\text {aux }}$. Also, the auxiliary system can be turned on as a current drive to achieve the necessary $I_{p}$ in the fully non-inductive cases, where $V_{\text {loop }}$ is constrained to zero. In the inductive cases however, a simultaneous presence of xenon and NBI is excluded by the control scheme. The upper limit on $P_{\text {sep }}$ is set essentially to protect the divertor, whereas the lower limit is introduced in order to ensure that a detrimental HL back transition be avoided.

The second requirement is fulfilled by controlling the argon concentration in the SOL. Thus, if for example the temperature at the plate $T_{p l}$ calculated with the $0 \mathrm{D}$ divertor model is above $5 \mathrm{eV}$, ASTRA increases the concentrations of argon in SOL and divertor region $\left(c_{z, A r}^{S O L}\right.$ and $\left.c_{z, A r}^{d i v}\right)$ until it drops below the threshold value, reducing the concentrations otherwise (ASTRA tries to minimise $c_{z, A r}^{S O L}$, also). The increase of argon in the SOL and in the divertor region is expected to lead, as a side effect, to an increase of the argon concentration in 
the core as well, therefore reducing the fusion power outcome via fuel dilution and via radiative losses. In the present work, it is assumed that a constant compression factor exists for argon, i.e. the concentration of argon in the SOL and in the divertor is at every timestep a factor $f_{c}^{A r}$ larger than the one in the core,

$$
c_{z, A r}^{S O L}=c_{z, A r}^{d i v}=f_{c}^{A r} c_{z, A r}^{C o r e} .
$$

with $f_{c}^{A r}$ set to 6 . A specular constraint has been put on Xe, with $f_{c}^{X e}=1 / 6$, as it is supposed to be injected directly in the core and not puffed in the divertor (the role of Xe as a SOL radiator can however be shown to be marginal). This is of course a quite crude approximation, as it assumes an instantaneous propagation of any change in the impurity concentration. Investigating the possibility of confining the radiating impurities in the SOL with such compression factors is a point of greatest interest in the current tokamak research, but this goes way beyond the purposes of the present paper. Incidentally, the effect of edge localised modes (ELMs) both on the impurity transport at the separatrix (they are expected to flush impurities from the core, allowing for higher $f_{c}^{A r} \mathrm{~s}$ ) and on the divertor load (which can increase dramatically, destroying the detachment) has not been adressed in this work.

ASTRA simulates the time evolution of the kinetic plasma profiles and of the magnetic equilibrium through a Grad-Shafranov solver. Each simulation is performed until convergence of the profiles, ensuring that the requirements on $P_{L H}$ and on the divertor heat flux are fulfilled and the imposed value of the $\mathrm{H}$ factor, see appendix I, is met.

\subsection{Scan parameters}

We have performed various scans in $R$ and $B_{T}$, varying the former between 2 and $18 \mathrm{~m}$ and the latter between 2 and $20 \mathrm{~T}$. In particular, we have repeated the scan for

- Inductive cases, with $q=3$ and $V_{\text {loop }}$ being an output,

- Non-inductive cases, where $q=3$ and 4 and $V_{\text {loop }}$ is set to the value of 0 . This corresponds to steady-state tokamak scenarios, with infinite pulse length and no use of the central solenoid during the flat-top phase.

Note that the safety factor $q$ has to be understood as a proxy for $q_{95}$ as no separatrix is modelled. The aspect ratio $A$ has been given the values of 2, 2.6. 3.1 , 4, whereas the $\mathrm{H}$ factor has been varied between 0.85 and 1.22 , playing however the role of control knob rather than of additional free parameter (i.e. not all possible combinations of $R, B_{T}$ and $\mathrm{H}$ have been considered, mainly because not every point turns out to be stable for every value of $\mathrm{H}$ ).

In the following sections, unless where explicitely mentioned, the shown results refer to inductive cases with $\mathrm{H}=1$ and $A=3.1$, as the most important 
conclusions we want to highlight are qualitatively independent on $\mathrm{H}, A$ and $V_{\text {loop }}$. In section 3.5, some considerations on the role of these parameters are reported.
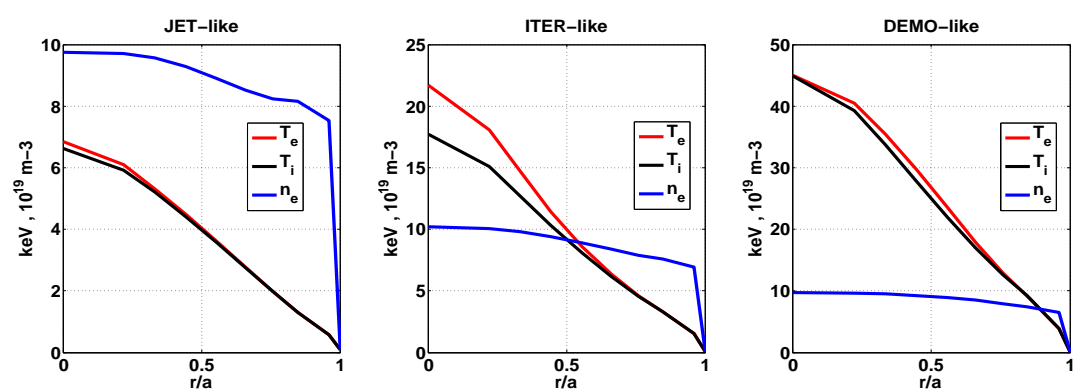

Figure 1: Three examples of converged density and temperature profiles in ASTRA as a function of the normalised minor radius coordinate. The "JET-like" machine has $R=3.67 \mathrm{~m}$ and $B_{T}=3 \mathrm{~T}$, the "ITER-like" has $R=6.3 \mathrm{~m}$ and $B_{T}=4.7 \mathrm{~T}$ whereas the "DEMO-like" is located by $R=9.1 \mathrm{~m}$ and $B_{T}=6.4 \mathrm{~T}$.
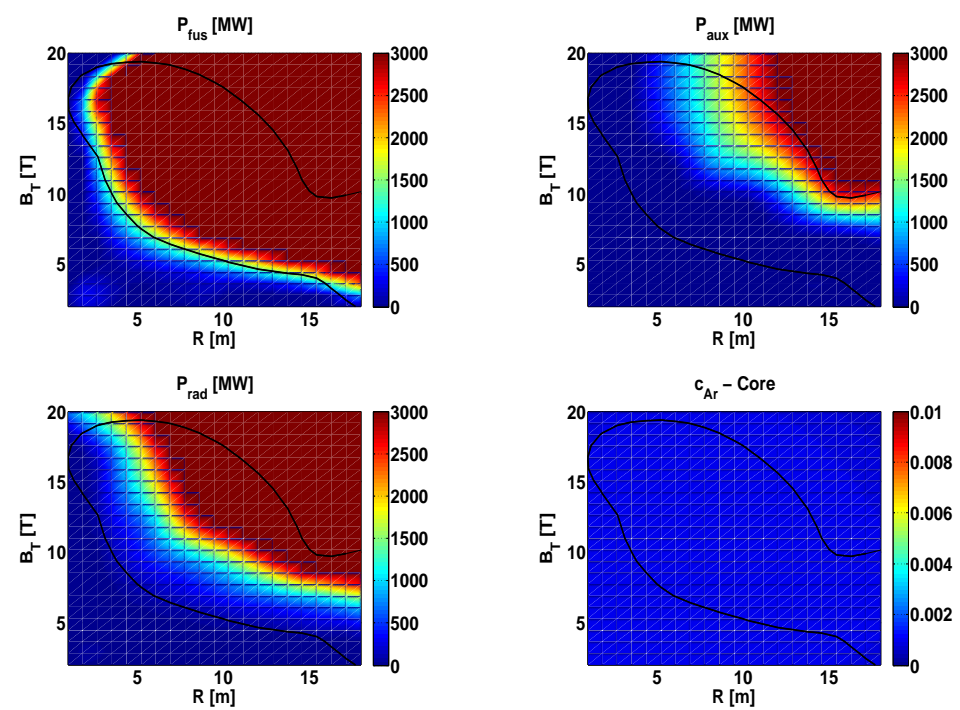

Figure 2: ASTRA results for Xe only cases (i.e. no divertor compatibility enforced). From the upper left corner clockwise: fusion power, auxiliary power, Ar concentration (which is obviously zero everywhere) and radiated power as a function of $R$ and $B_{T}$. The black lines correspond to the $P_{e l}=500 \mathrm{MW}$ contour, see Fig.3 below. For these simulations, we have set $\mathrm{H}=1$ and $q=3$ (inductive machines). 


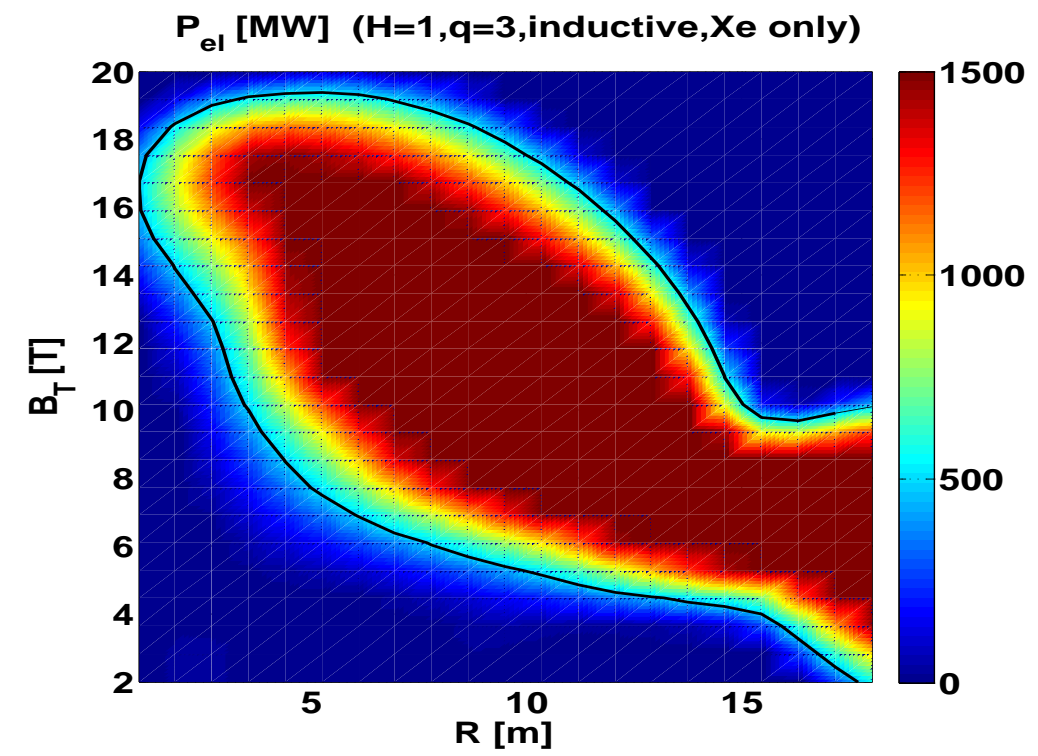

Figure 3: Net electrical power for Xe only cases (i.e. no divertor compatibility enforced). The black line highlights the $P_{e l}=500 \mathrm{MW}$ level curve. For these simulations, we have set $\mathrm{H}=1$ and $q=3$ (inductive machines). The "opening up" of the curve at low $B_{T}$ and high $R$ is due to the favourable scaling of $\tau_{E}$ with $R$, which reduces the necessary $P_{a u x}$.

\section{Results and discussion}

As previously mentioned, ASTRA is a $1.5 \mathrm{D}$ code which calculates in a selfconsistent way the most relevant tokamak parameters as a function of the radial coordinate, together with a Grad-Shafranov solver to determine the structure of the magnetic surfaces. An example of ASTRA output profiles is shown in Fig.1, where a "JET-like", a "ITER-like" and "DEMO-like" case (where "like" means with $R$ and $B_{T}$ of the same order as the indicated machine) are compared. It is incidentally interesting to note that the three devices mainly differ in temperature, their density values being otherwise very close to each other.

\subsection{Xe-only Case}

We start to present the ASTRA results obtained without employing the OD model to determine the divertor conditions. This means that the divertor is supposed to sustain the incoming exhaust power without any repercussion on the core. Under these assumptions, the use of argon is unnecessary, and therefore neglected (or, equivalently, we assume Ar to be perfectly confined in the SOL independently of its concentration). The constraint $P_{\text {sep }}<1.2 P_{L H}$ is 

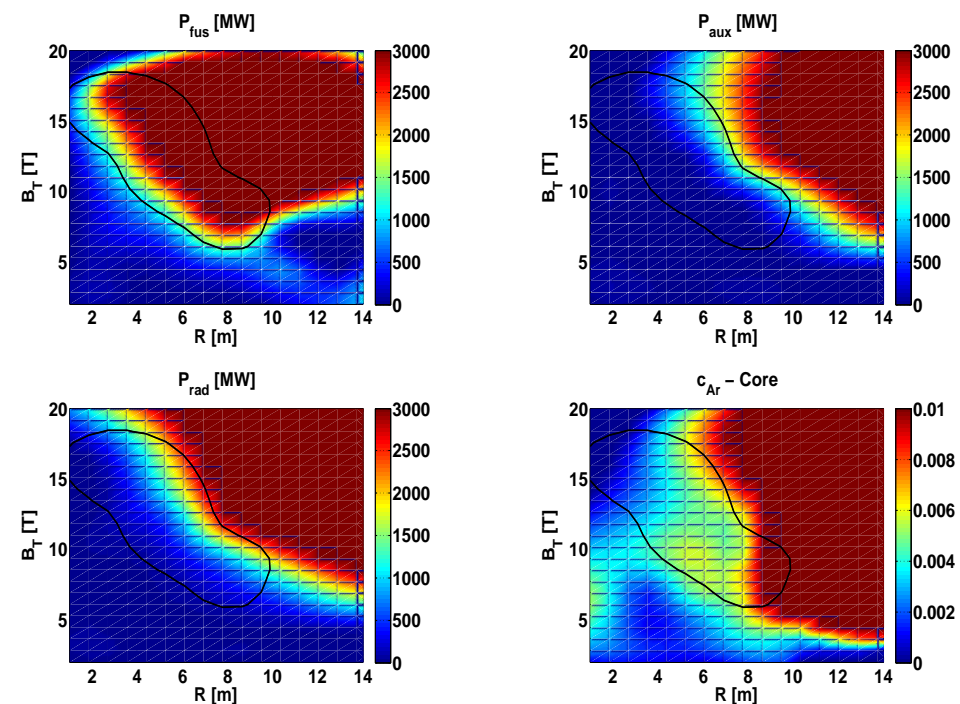

Figure 4: ASTRA results for Xe+Ar cases (i.e. with divertor compatibility enforced). From the upper left corner clockwise: fusion power, auxiliary power, Ar concentration and radiated power as a function of $R$ and $B_{T}$. The black lines correspond to the $P_{e l}=500 \mathrm{MW}$ contour, see Fig. 5 below. For these simulations, we have set $\mathrm{H}=1$ and $q=3$ (inductive machines).

ensured by means of Xe, whereas its counterpart $P_{\text {sep }}>1.1 P_{L H}$ is, where necessary, guaranteed through the use of NBI power.

Fig.2 shows the fusion power, the auxiliary power and the radiative losses as a function of $R$ and $B_{T}$ for inductive cases with $q=3$ and $\mathrm{H}$-factor set to 1 . As expected, the fusion power grows both with $R$ and $B_{T}$. However, at very high $R$ and $B_{T}$, the synchrotron radiation also reaches quite high values, dramatically enhancing the radiative losses (the presence of $P_{\text {aux }}$ for those points let us conclude that no line radiation from Xe is present). In order to avoid an excessive cooling of the plasma, a massive injection of auxiliary power becomes necessary. One can imagine to estimate the net electric power $P_{e l}$ a machine can produce as the difference between the outcome of the thermodynamic cycle and the recirculating power, namely

$$
P_{e l}=\left(P_{\text {fus }}+P_{a u x}\right) \eta_{t h}-\frac{P_{a u x}}{\eta_{w p}}
$$

where $\eta_{t h}$ is a thermodynamic efficiency and $\eta_{w p}$ is the wall-plug efficiency of the auxiliary. In this simple analysis, only $P_{a u x}$ has been considered in the evaluation of the recirculating power, other power losses being for the moment ignored. Also, the contribution to the thermodynamic cycle from the heat 


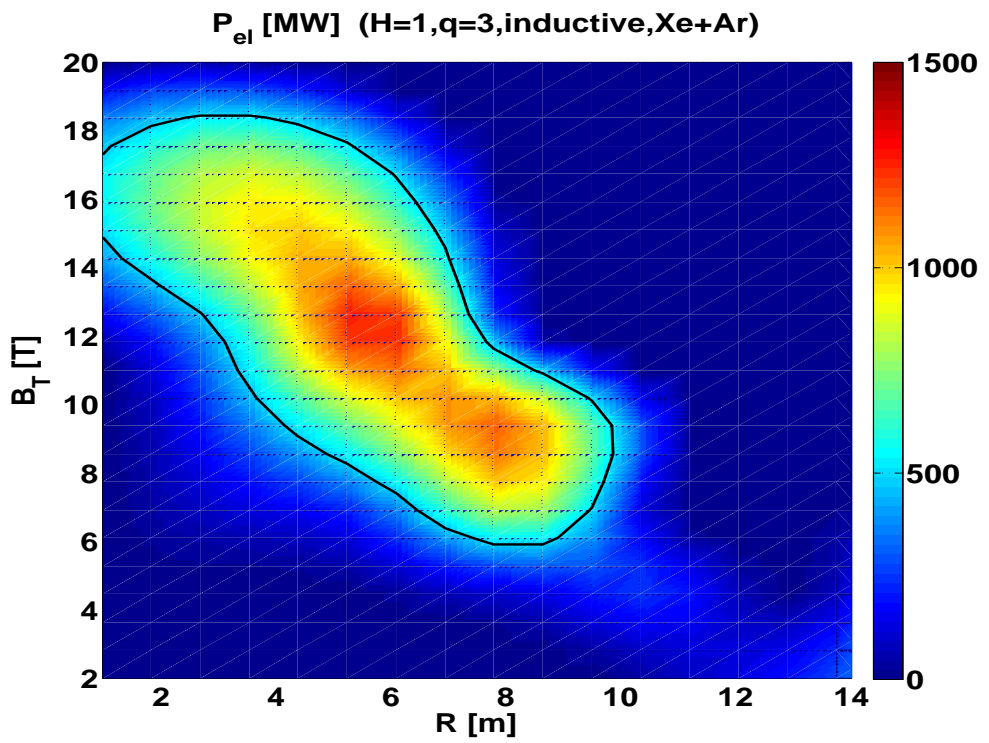

Figure 5: Net electrical power for $\mathrm{Xe}+\mathrm{Ar}$ cases (i.e. with divertor compatibility enforced). The black line highlights the $P_{e l}=500 \mathrm{MW}$ level curve. For these simulations, we have set $\mathrm{H}=1$ and $q=3$ (inductive machines).

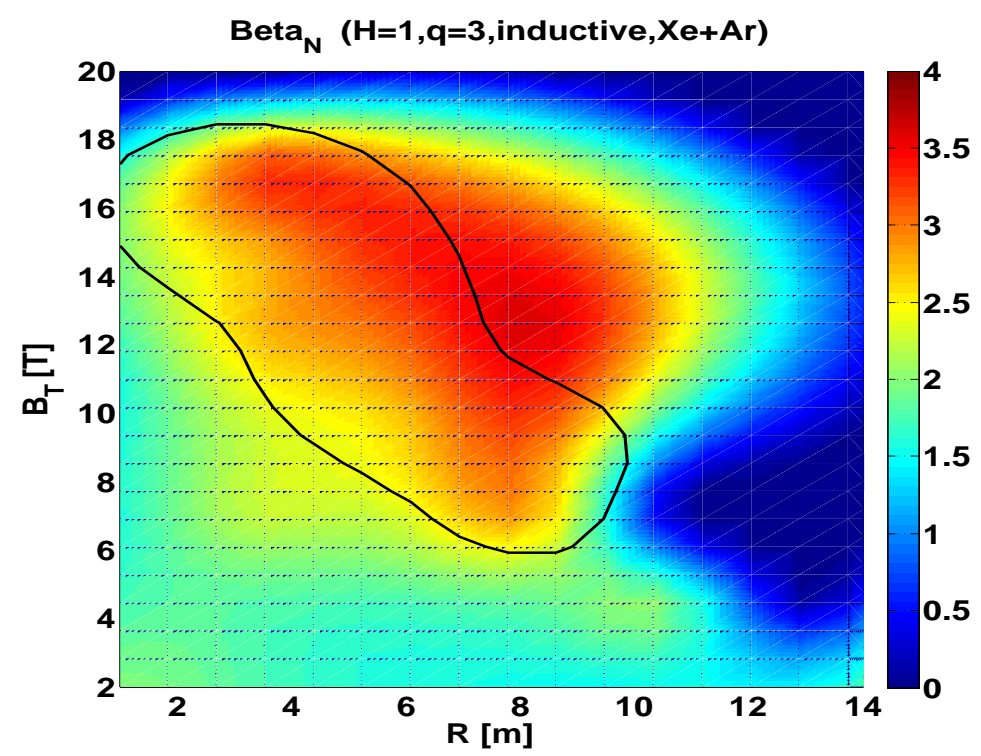

Figure 6: Value of the pressure parameter $\beta_{N}=\beta\left(a B_{T}\right) / I_{p}$ for the same points as in Fig.5. The black line highlights the $P_{e l}=500 \mathrm{MW}$ level curve. 

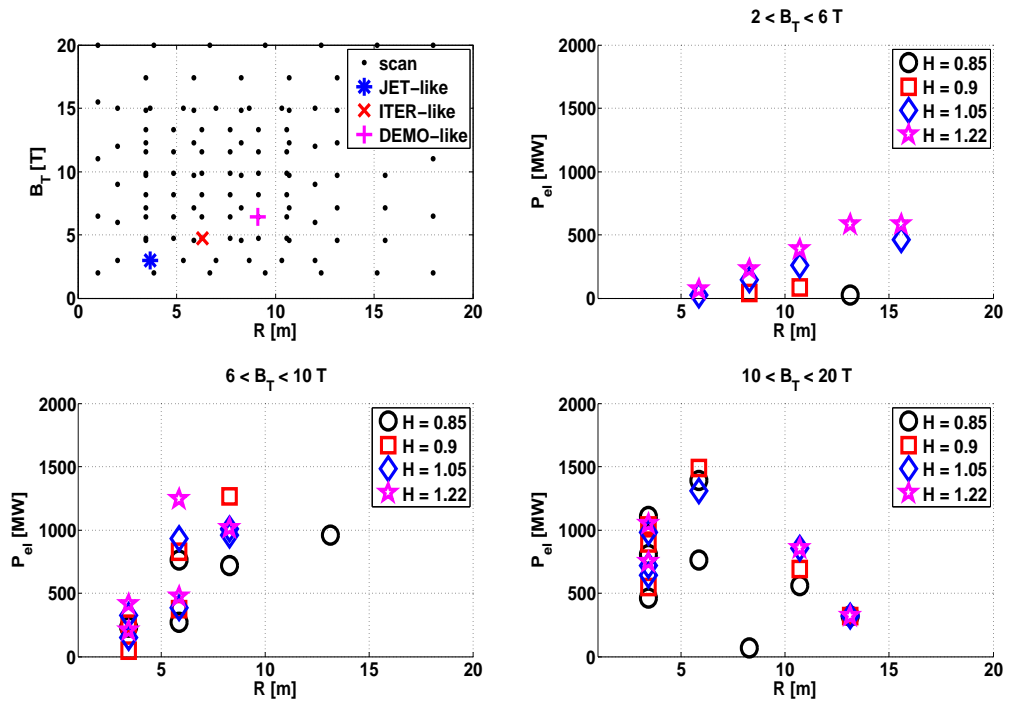

Figure 7: $\mathrm{H}$ factor scan. Upper left corner: $R$ and $B_{T}$ points considered in the scan. As a reference, the three points depicted in Fig.1 are represented. The remaining three plots show the net electrical power for the points in the upper left corner as a function of $R$ and of the imposed $\mathrm{H}$ factor for different intervals of $B_{T}$ values. Points at negative electric power are not shown.

generated by nuclear reactions for tritium breeding in the blanket has been neglected. Fig.3 shows the value of $P_{e l}$ for the same points as in Fig.2. A first conclusion we can draw is that there clearly exists a limit beyond which further increases in $R$ or $B_{T}$ deteriorate the maximum achievable electricity production of the tokamak instead of improving it. This occurrence can be explained by analysing the dependence of $P_{\text {fus }}$ and $P_{\text {sync }}$ on $R$ and $B_{T}$. The fusion power per unit volume grows almost quadratically with the temperature at around 20 $\mathrm{keV}$, this dependence being however quite weakened when $T \gg 20 \mathrm{keV}$. On the contrary, the synchrotron radiation has a stronger dependence on both $B_{T}$ and the temperature, see Eq.5, thus it grows quickly when the machine becomes sufficiently large (we recall that an increase in $R$ mainly reflects in an increase in $T$, see Fig.1). As the fusion power contributes to the electrical power being multiplied by a factor smaller than one, whereas $P_{\text {sync }}$ represents a loss which has to be compensated by $P_{a u x}$, which is divided by a factor smaller than one, it is clear that at some point any increase in $P_{\text {fus }}$ will be annihilated by a corresponding increase in the synchrotron radiation. In the present analysis, the values

$$
\begin{gathered}
\eta_{t h}=0.35 \\
\eta_{w p}=0.4
\end{gathered}
$$




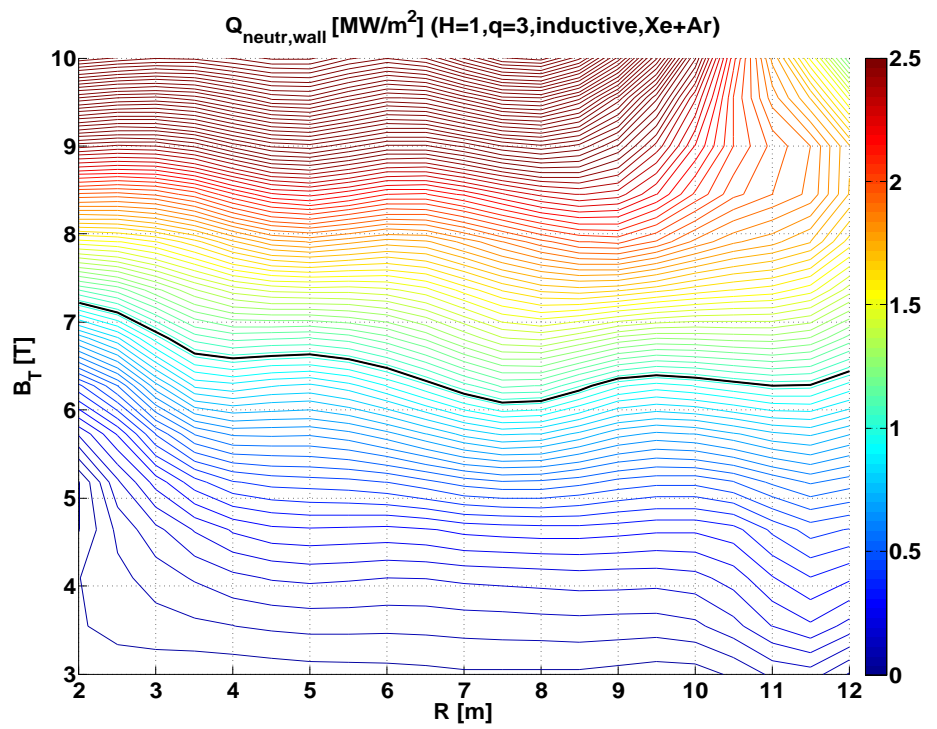

Figure 8: Estimated neutron flux $Q_{\text {neutr,wall }}$ at the first wall for the ASTRA points in Fig.5 as a function of $R$ and $B_{T}$. The curves are approximatively horizontal as both the fusion power and the first wall surface grow almost quadratically with the major radius. The curve at $Q_{\text {neutr,wall }}=1 \mathrm{MW} / \mathrm{m}^{2}$ is highlighted in black.

(which are close to the values currently employed for the european DEMO 1 [20]) have been chosen, but it is important to stress that a change in the numerical value of these parameters would not lead to a qualitatively different plot, because there will always exist a limit beyond which the increase in fusion power is overcompensated by a corresponding increase in the radiative losses. This limit is clearly a hard physical limit, fairly independent on any achievable technological improvement we may be aiming at.

The region where $P_{e l}>500 M W$, delimited by the black curve in Fig.3, has approximatively the shape of an hyperbola, which does not close on itself even at very large radii. This can be explained because the synchrotron radiation scales as $\left(B_{T} T\right)^{2.5}$, see Eq.5, thus it is necessary to decrease $R$ (and therefore $T$ ) to increase $B_{T}$ without increasing $P_{\text {sync }}$, and viceversa. We can therefore state that a large machine requires a weak magnetic field to produce a reasonable amount of electrical power. The converse statement, i.e. that a small machine can be operated at larger magnetic fields, needs however a bit more prudence and will be discussed in more detail in the following. It is however important to stress that the pulse length corresponding to each scan point, which is a crucial parameter to evaluate the effective performance of a pulsed device like a tokamak, has for the moment been left outside our analysis. We expect however the scenarios at high $B_{T}$ and low $R$ to be unfavourable from 


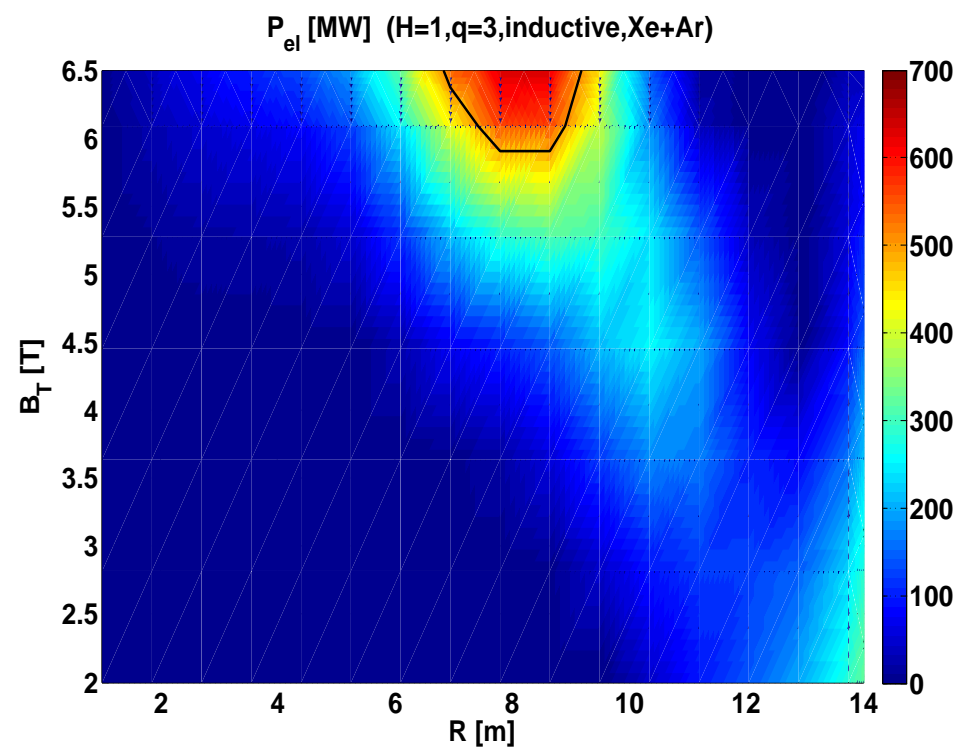

Figure 9: Same as Fig.5 enforcing the technological condition $B_{T}<6.5$ T. Note that the color scale is not the same as in the previous case.

this point of view, both because of the limited size of the central solenoids they can contain and for their higher $V_{\text {loop }}$.

\subsection{Xe-Ar Case}

In this second subsection, the 0D model is coupled to ASTRA and the divertor protection as depicted in section 2 is taken into account. Now, argon is also introduced in the system with the purpose of bringing the temperature at the divertor plates at sufficiently low values. The argon injected in the system does not remain confined in the SOL, but it also dilutes the core, deteriorating therefore the performance of the machine. We recall we have assumed a constant ratio between the relative concentration of Ar in the SOL and in the core.

The impact of the divertor related constraint on the production of energy can be visualized in Fig.4 and 5. The effect of argon is much more relevant at higher $R$ rather than at higher $B_{T}$, this being essentially linked to the fact that $P_{L H}$ depends more strongly on $R$ than $B_{T}$, see Eq.10, and thus the magnitude of the power exhaust which has to be radiated before reaching the target plate is more sensitive on the machine size rather than on the field. This occurrence has been already pointed out in a recent work of Reinke [21], where the following scaling for the impurity concentration in the SOL to reach detachment 
$c^{\text {Reinke }}$, based on a simple 0D argument, has been suggested:

$$
c^{\text {Reinke }} \propto B_{T}^{0.88} R^{1.33} .
$$

The author used that relation to support the idea that, for the divertor protection, compact machines at higher field are more efficient, in agreement with what our investigation has pointed out. The effect on the achievable net electric power, reported in Fig.5, is thus that the curves at constant $P_{\text {el }}$ become close on themselves, as the power outcome is limited by divertor protection requirements (i.e. radiating impurities in the SOL) at high $R$ and low $B_{T}$, whereas the radiative losses, as pointed out in the previous section, reduce the achievable power production at high $R$ and $B_{T}$. The fact that the level curves of $P_{e l}$ are closed on themselves implies that a maximum achievable electric power exists at intermediate values of radius and magnetic field. In the presented figures, this maximum is located at around $R=6 \mathrm{~m}$ and $B_{T}=13 \mathrm{~T}$. Clearly, these numbers have no intrinsic physical meaning, as they strongly depend on the choice of the arbitrary parameters contained in our model - it is straightforward to check how strong is the impact of $\eta_{t h}$ and $\eta_{w p}$ on the final $P_{e l}$ outcome, for example. Nevertheless, the existence of a maximum power level, independently on the technology employed, is a physical result of general validity for all fusion devices requiring a radiative $S O L$ and a minimum value for $P_{\text {sep }}$.

For completeness, in Fig.6 we also show the value of the normalised quantity $\beta_{N}$, which is an important indicator for the MHD stability of a given plasma equilibrium. As one can observe, the values we found lie mostly below the typical ideal wall limit of 3.5 for the external kink modes, although the presence of slowly growing resistive wall modes (RWM) cannot be excluded (see for example [22] for a review on the topic).

\subsection{The role of the $\mathrm{H}$-factor}

All the results presented so far referred to points with an $\mathrm{H}$ factor of 1 , so to isolate the influence of that quantity on the final outcome. However, if one considers also the points with $\mathrm{H}$ varying from 0.85 up to $\mathrm{H}=1.22$, the results emerging are quite interesting and, to some extent, counterintuitive. These are shown in Fig.7. On the upper left plot, the points considered for the scan are plotted on the $R-B_{T}$ plane, having highlighted also the three points whose profiles are represented in Fig.1 as a reference. On the other three plots, the corresponding electrical power outcome as a function of $R$, having sorted and grouped the points with respect to $B_{T}$ and $\mathrm{H}$, is shown. At low fields (upper right plot), it is immediate to see that an increase of $\mathrm{H}$ reflects on an improvement of the machine performance, as the star marker, identifying the highest $\mathrm{H}$-factor, is always above the others. Viceversa, at higher field (the two lowest plots), an enhancement in $\mathrm{H}$ does not necessarily lead to a larger electrical outcome, and actually one can notice that the highest electrical production is obtained for $B_{T}$ between 10 and $20 \mathrm{~T}$ at $R \sim 6 \mathrm{~m}$ with $\mathrm{H}$ lower than 1 (lower 
right corner). This to some extent unexpected effect is once again linked to the synchrotron radiation, as an increase of the energy confinement capability of the device implies an increase in the central temperature, which in certain regions of the $R-B_{T}$ plane leads to an increase of the radiative losses which cannot be compensated by the gain in fusion power. This result is in some sense in line with what has been concluded in the previous section, namely that the best performance in terms of electrical energy production has not to be looked for at extreme values of the confinement capability of the machine (very large field and radius), but rather that, even from the point of view of plasma physics only, the optimum choice is characterised by intermediate values of those quantities together with a moderate confinement capability.

\subsection{Technological considerations}

From the point of view of plasma physics, we have shown that for the production of electrical energy it is more convenient, up to a certain extent, aiming at higher fields rather than at bigger devices because of the divertor protection requirement. If one however introduces some technological constraints on top of the analysis, the scenario changes significantly. The discussion presented in this subsection is by no means complete, but it is useful to put in the right perspective some perhaps misleading conclusions which could be drawn by looking at the plasma physics side only.

An important technological aspect in the tokamak engineering is represented by the neutron load onto the first wall $Q_{\text {neutr,wall }}$, defined as

$$
Q_{\text {neutr }, \text { wall }}=\frac{4}{5} \frac{P_{f u s}}{S_{F W}},
$$

where $S_{F W}$ is the first wall surface. For the European DEMO 1, $Q_{\text {neutr,wall }}$ lies around $1 \mathrm{MW} / \mathrm{m}^{2}$, as reported in [20]. This value cannot increase too much, as the neutron fluence on the structural materials, see for example [14], limits the lifetime of the blanket components and therefore of the whole machine. Taking the DEMO 1 value as an upper limit, we see from Fig.8 that this condition is more or less equivalent to impose $B_{T}<6.5 \mathrm{~T}$, optimistically rounded, as the curves at constant $Q_{\text {neutr,wall }}$ are approximatively horizontal in the $R-B_{T}$ plane (because both $P_{f u s}$ and the first wall surface are proportional to $R^{2}$ ). Fig.9 shows the remaining feasible points once this additional requirement is enforced. As one can see by comparing Fig.9 with Fig.5, the vaste majority of points with $P_{e l}>500 \mathrm{MW}$ lie beyond such $Q_{\text {neutr,wall }}$ threshold, and, in particular, all the compact devices with high fields possess much higher values, this implying a much higher deterioration rate for the structures. Incidentally, the same argument concerning $Q_{\text {neutr,wall }}$ can be also applied to the total (electromagnetic) radiation power, which also exhibits a maximum tolerable limit per surface unity [23]. As the total radiation power (core plus SOL) can also be 
assumed to be roughly proportional to the achieved fusion power (only a very tiny fraction of the power actually reaches the divertor plates), similar curves as in Fig.8 are expected to appear, reinforcing the conclusions herewith presented.

Clearly, the surface load is only one example of the technological constraints which can be imposed, although, probably, one of the most critical. Other aspects, as for example the feasibility of plasma fueling as investigated in [24], or the already mentioned achievable pulse length, lie beyond the scope of the present manuscript.

\subsection{Non-Inductive points and effects of A}

The results which have been presented up to now - and in particular, the fact that the curves at constant $P_{e l}$ are closed on themselves - are absolutely general, and do not qualitatively depend on the chosen value of $q, V_{\text {loop }}$ or $A$. In this section however, we will comment about the sensitivity of the electric power production on such parameters.

\begin{tabular}{|c|c|c|c|c|}
\hline $\mathbf{H}$ & $\mathrm{Xe}, q=3$ & $\mathrm{Xe}+\mathrm{Ar}, q=3$ & $\mathrm{Xe}+\mathrm{Ar}, q=4$ (st.) & $\mathrm{Xe}+\mathrm{Ar}, q=3$ (st.) \\
\hline 0.9 & 801.3 & 658.9 & $\mathrm{x}$ & $\mathrm{x}$ \\
\hline 1.0 & 1013.2 & 766.6 & 68.9 & 119.9 \\
\hline 1.2 & 1255.0 & 985.1 & 115.3 & 285.3 \\
\hline 1.4 & $\mathrm{x}$ & $\mathrm{x}$ & 205.0 & 325.7 \\
\hline
\end{tabular}

Table 1: Variation of $P_{e l}$ for different combinations of $q, V_{l o o p}$ and H. Columns marked with (st.) indicate steady-state tokamak configurations, i.e. $V_{\text {loop }}=0$. The values of $P_{e l}$ are given in MW. The considered machine has $R=9.1 \mathrm{~m}$ and $B_{T}=6.4$ $\mathrm{T}$. Cells containing an " $\mathrm{x}$ " indicate non-converged points.

In table 1, the calculated $P_{e l}$ for a machine with $R=9.1 \mathrm{~m}$ and $B_{T}=6.4$ $\mathrm{T}$ (i.e. the "DEMO-like" device of Fig.1) for different $\mathrm{H}$ factors, $q$ and $V_{\text {loop }}$, are reported. As one can immediately notice, the points are found in a region of the parameter space where an increase of the confinement factor $\mathrm{H}$ reflects in a better performance in terms of electricity production. For the same $\mathrm{H}$ value, steady-state machines (i.e. $V_{\text {loop }}=0$ ) exhibit a much lower electricity output if compared to the corresponding pulsed device, as the need of obtaining the necessary plasma current through external sources significantly impacts on the recirculating power fraction. The situation improves partially at higher $\mathrm{H}$ factors, as the higher $\beta$ allows a larger bootstrap current fraction, limiting therefore the magnitude of the necessary current drive (the fact that a high $\mathrm{H}$ is needed for operating a steady-state device is consistent with the results published in [12]). It is interesting to note that, in this particular region of the parameter space, a steady-state machine with $q=3$ has a better performance than a $q=4$ device, because the growth in $P_{f u s}$ is sufficient to overcompensate the costs of an increased current drive. To our knowledge however, no advanced scenarios with such a low safety factor have been up to now experimentally observed. 


\begin{tabular}{|c|c|}
\hline $\mathbf{A}$ & $P_{e l}[\mathbf{M W}]$ \\
\hline 3.1 & -47.3 \\
\hline 2.6 & 0.0 \\
\hline 2.0 & 1510.5 \\
\hline
\end{tabular}

Table 2: Variation of $P_{e l}$ as a function of $A$ for a non-inductive machine with divertor protection enforced, $q=3, R=4.42 \mathrm{~m}$ and $B_{T}=6.29 \mathrm{~T}$.

In table 2, the impact of the variation of the aspect ratio $A$ on a small, noninductive device are illustrated. This choice is justified by the fact that, for compact devices, the effect of the aspect ratio is much more significant than on the big machines. Furthermore, the steady-state operation allows to ignore the problem of the size of the central solenoid, which is on the contrary of primary importance for pulsed machines with low aspect ratio. As one can observe, the impact of such variable on the reactor performance is indeed dramatic - its main effect being linked to a volume increase when $R$ is kept constant. It is however necessary to stress that no conclusions on the actual feasibility of a low aspect ratio device can be drawn by looking at this table only, as it has to be understood as a simple sensitivity analysis. For example, a decrease of $A$ for a given $B_{T}$ leads to an increase of the field on the toroidal field coils, which is limited by technological considerations. Furthermore, the quantity $\beta_{N}$ for $A=2$ largely exceeds the value of 4 , letting us conclude that the corresponding operational point is most probably MHD unstable and thus not appropriate for a fusion reactor.

\subsection{Effect of $\tau_{E}$ scaling law}

In the present work, the energy confinement time $\tau_{E}$ has always been calculated by employing the well-known IPB98(y,2) scaling [9, 10]. The corresponding $\tau_{E, I P B 98(y, 2)}$ exhibits the following dependencies

$$
\tau_{E, I P B 98(y, 2)} \propto P_{\text {out }}^{-0.69} R^{1.9}
$$

where

$$
P_{\text {out }}=P_{\text {sep }}+P_{\text {rad }, \text { core }}
$$

and $P_{\text {rad,core }}$ is the total radiation power from inside the separatrix. A known problem of such scaling is that it refers to a database of experimental points with low $P_{\text {rad,core }}$. At present, the validity of such scaling for the highly radiative, reactor relevant scenarios is therefore extremely debated. Without entering in the details of the discussion, which is still open and ongoing in the fusion community, we limit ourselves to introduce here an alternative, radiation corrected IPB98 $(\mathrm{y}, 2)$ confinement time defined as

$$
\tau_{E, I P B 98(y, 2)}^{\text {Rad.Corr. }}=\tau_{E, I P B 98(y, 2)}\left(\frac{\hat{P}_{\text {out }}}{P_{\text {out }}}\right)^{-0.69}
$$


where

$$
\hat{P}_{\text {out }}=P_{\text {sep }}+P_{\text {rad,core }}^{\rho_{p}>0.6}
$$

$P_{\text {rad,core }}^{\rho_{p}>0.6}$ being the fraction of $P_{\text {rad,core }}$ radiated from outside the magnetic surface corresponding to $\rho_{p}=0.6$, where $\rho_{p}$ is the dimensionless magnetic flux coordinate defined as

$$
\rho_{p}=\sqrt{\frac{\psi-\psi_{s e p}}{\psi_{0}-\psi_{s e p}}}
$$

$\psi$ is the magnetic flux and the subscripts 0 and sep indicate the magnetic axis and the separatrix, respectively.

The IPB98(y,2) is not the only confinement time scaling law which has been suggested, although by far the most employed. For example, a so called electrostatic gyro-Bohm scaling exists, which exhibits the following dependence on power and radius

$$
\tau_{E, E G B} \propto P_{\text {out }}^{-0.55} R^{2.1}
$$

This scaling has been produced on the assumption that the energy transport is dominated by electrostatic turbulence following the gyro-Bohm scaling. More details on this can be found in [25].

In this section, we investigate on three different machines $(R=5.86,8.29$ and $10.71 \mathrm{~m}$ for $B_{T}=7.14$, inductive, with $A=3.1, q=3$ and divertor protection enforced) the consequence of assuming the energy transport to be determined by each of the three confinement scalings elucidated above - having set $\mathrm{H}=$ $\tau_{E} / \tau_{E, \text { scaling }}=1$ for each scaling. The results are summarised in tables 3,4 and 5 . One can observe that, in general, when $\tau_{E}$ is determined via the electrostatic gyro-Bohm scaling, the corresponding profiles show a larger synchrotron loss than IPB98 $(\mathrm{y}, 2)$ as a consequence of the increase of the temperatures, this occurrence being particularly visible for the largest $R$. In other words, if the energy transport physics would follow what the electrostatic gyro-Bohm scaling assumes, the more favorable size dependence of $\tau_{E}$ would reflect in a worse electric power at larger radii. This is equivalent to what has been concluded in section 3.3, namely that an improved confinement not necessarily leads to a better performance in terms of $P_{e l}$, but rather that, in the high temperature regions of the parameter space, this might have detrimental effects.

\begin{tabular}{|c|c|c|c|}
\hline$\tau_{E}$ scal. law & $P_{e l}[\mathrm{MW}]$ & $P_{\text {fus }}[\mathrm{MW}]$ & $P_{\text {sync }}[\mathrm{MW}]$ \\
\hline IPB98(y,2) & 1160.8 & 3095.5 & 125.4 \\
\hline EGB & 1096.4 & 2923.8 & 343.2 \\
\hline Rad.Corr. & 663.0 & 1768.0 & 89.2 \\
\hline
\end{tabular}

Table 3: Variation of $P_{e l}, P_{f u s}$ and $P_{\text {sync }}$ having set $\mathrm{H}=1$ for different $\tau_{E}$ scalings. The data refer to a machine with $R=8.29 \mathrm{~m}$ and $B_{T}=7.14 \mathrm{~T}$ (with Ar, $q=3$, inductive and $A=3.1$ ). 
We note also that the points calculated assuming the radiation corrected IPB98(y,2) scaling possess a lower electric power yield in comparison to the non radiation corrected. This depends on the fact that the confinement time is longer, but the energy stored in the plasma $W$, which determines the fusion power outcome, is on the contrary lower (as $W \sim P_{\text {out }} \tau_{E} \sim P_{\text {out }}^{0.3}$, but for the radiation corrected case one assumes consistently $W \sim \hat{P}_{\text {out }}^{0.3}$ ).

\begin{tabular}{|c|c|c|c|}
\hline$\tau_{E}$ scal. law & $P_{e l}[\mathrm{MW}]$ & $P_{\text {fus }}[\mathrm{MW}]$ & $P_{\text {sync }}[\mathrm{MW}]$ \\
\hline IPB98(y,2) & 557.3 & 1486.2 & 29.8 \\
\hline EGB & 488.9 & 1303.8 & 77.2 \\
\hline Rad.Corr. & 266.8 & 711.5 & 21.4 \\
\hline
\end{tabular}

Table 4: Variation of $P_{e l}, P_{f u s}$ and $P_{\text {sync }}$ having set $\mathrm{H}=1$ for different $\tau_{E}$ scalings. The data refer to a machine with $R=5.86 \mathrm{~m}$ and $B_{T}=7.14 \mathrm{~T}$ (with Ar, $q=3$, inductive and $A=3.1$ ).

\begin{tabular}{|c|c|c|c|}
\hline$\tau_{E}$ scal. law & $P_{e l}[\mathrm{MW}]$ & $P_{f u s}[\mathrm{MW}]$ & $P_{\text {sync }}[\mathrm{MW}]$ \\
\hline IPB98 $(\mathrm{y}, 2)$ & 1848.4 & 4929.1 & 337.9 \\
\hline EGB & 711.7 & 4464.2 & 1028.4 \\
\hline Rad.Corr. & 1247.6 & 3327.0 & 237.0 \\
\hline
\end{tabular}

Table 5: Variation of $P_{e l}, P_{f u s}$ and $P_{s y n c}$ having set $\mathrm{H}=1$ for different $\tau_{E}$ scalings. The data refer to a machine with $R=10.71 \mathrm{~m}$ and $B_{T}=7.14 \mathrm{~T}$ (with $\mathrm{Ar}, q=3$, inductive and $A=3.1$ ).

The correct understanding of the confinement time behaviour for different machine parameters has been shown here once more to be a very important feature in view of the realisation of the future nuclear fusion reactors.

\subsection{Analysis of the ST Pilot Plant}

The formula we employed to determine the net electric power production, Eq.12, can be easily re-written as

$$
P_{e l}=P_{a u x}\left[\frac{(Q+1) \eta_{t h} \eta_{w p}-1}{\eta_{w p}}\right]
$$

by straightforward algebra. Here, the widely employed fusion gain parameter $Q=P_{f u s} / P_{a u x}$ has been introduced. Necessary condition for a fusion machine to produce a positive electricity output is therefore

$$
Q>\frac{1}{\eta_{t h} \eta_{w p}}-1 .
$$

For higher values of $\eta_{t h}$ and $\eta_{w p}$, the electricity production of a given plasma configuration increases at fixed $Q$ - as one could expect. The values we have employed for $\eta_{t h}$ and $\eta_{w p}$ in the current investigation are already quite optimistic. The value $\eta_{t h}=0.35$, for example, does not take into account all the 
recirculating power in the balance of plant, and more in general all the parasitic losses in the mechanical components. Typical DEMO 1 scenarios [14] refer to an electricity production of $500 \mathrm{MW}$ for a fusion power of around $2 \mathrm{GW}$, which in our approach would mean $\eta_{t h} \sim 0.25$ ( $\eta_{t h}$ being in this case understood as a "global" efficiency, rather than a purely thermodynamical constraint). Also, the value of $\eta_{w p}=0.4$ is quite high for the current technological status. These two values, when plugged in Eq.24, lead to $Q>6.14$ as a necessary condition for the net electric power to be positive.

The machine suggested by Costley and co-authors in a recent paper [17], called ST Pilot Plant, is a compact device $(R=1.35 \mathrm{~m})$ with low aspect ratio, a fusion power of $185 \mathrm{MW}$ and characterised by steady-state discharges, representing in the intention of the authors a device for electricity production. The most relevant parameters are summarised in table 1 of the cited paper, to which we refer the interested reader. In spite of the extremely optimistic assumptions the authors made in the course of their analysis (e.g. an $\mathrm{H}$ factor of 1.88 together with an helium fraction of $2 \%$ ), the device is characterised by a gain $Q=5$, as openly stated in the cited table 1. Thus, unless assuming unrealistically high values for $\eta_{t h}$ and $\eta_{w p}$ (on top of the other very favourable assumptions the paper contains), the machine proposed by Costley et al. cannot be regarded as a reliable option for the production of electrical power, as its fusion gain is not sufficient to guarantee the net electricity outcome to be positive. This conclusion, based on a very simple argument, has been confirmed by our tool, where a more accurate determination of the fusion power and of the necessary auxiliary power has been performed, yielding values for $P_{f u s}$ and $P_{a u x}$ of the same order as what reported in [17].

\section{Conclusions}

With a tool able to simultaneously model core transport and thermal power balance in the SOL, we have performed a large number of scans in the major radius $R$ and magnetic field $B_{T}$ for different values of aspect ratio, loop voltage and $\mathrm{H}$ factor, with the purpose of identifying the most attractive solutions for electricity producing tokamaks. The main result emerging from the present analysis is that, when plotted in the $R-B_{T}$ plane, the curves at constant electrical energy output are closed on themselves, and a maximum achievable power exists. Although the absolute numbers resulting from our calculation depend on the values we chose for the parameters in the model (such like $\eta_{t h}$ or the impurity compression factors), possessing therefore no general applicability, the result of closed $P_{e l}$ curves in the $R-B_{T}$ plane is universal for all magnetically confining devices which need a radiative SOL for the divertor protection and exhibit a lower limit on the power exhaust.

In particular, we found out that points at high $R$ and $B_{T}$ cannot provide a significant electricity outcome because the benefits of a higher fusion power 
are overcome by the necessity of large auxiliary power injections to compensate the large losses caused by synchrotron radiation. In contrast, at high $R$ and low $B_{T}$, the concentrations of impurities needed to detach the divertor are sufficiently high to strongly dilute the fusion fuel and therefore depleting the fusion power outcome. From the point of view of plasma physics only, compact devices at high $B_{T}$ and low $R$ are found to be more attractive, but from the technological point of view they can be shown, even with simple arguments, to be more problematic, as the reduced dimension causes higher load densities on the plasma facing components. Our calculations seem therefore to indicate that a machine of intermediate size and field is the optimum we can aim at for the production of electrical energy from nuclear fusion reactions with tokamaks, unless major technological progresses are made.

A deeper insight on the technological aspects of the problem, and also an estimate of the cost of electricity associated to the different reactor configurations represent the most natural prosecution of our work, which we leave for the future. Especially, an evaluation of the impact of the pulse length on the average electricity production, which makes the steady-state scenarios more attractive than what the present analysis suggests, is an important point that needs to be addressed. Also, a study by means of our tool of dynamical phenomena such as ramp-up and down is planned for the near future.

\section{Acknowledgment}

The authors would like to thank Dr. Fabio Subba for the DEMO SOLPS runs against which the 0D SOL model has been calibrated.
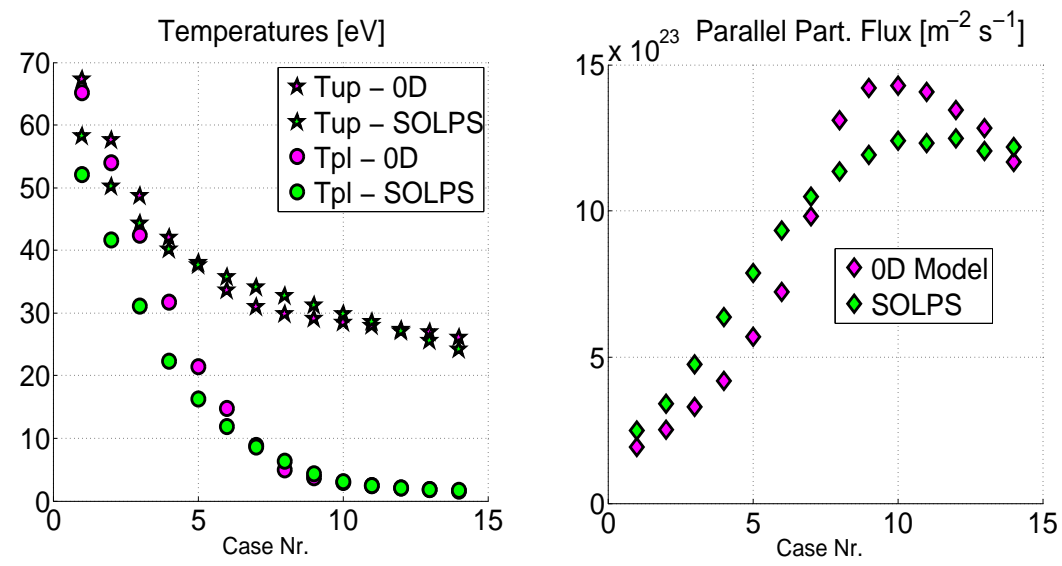

Figure 10: Comparison of the extreme temperatures $T_{u p}$ and $T_{p l}$ (left) and of the parallel particle flux $\Gamma_{\|, p l}$ (right) between the 0D model and SOLPS runs. The shown SOLPS runs refer to ASDEX Upgrade cases with intrinsic impurities (carbon). 


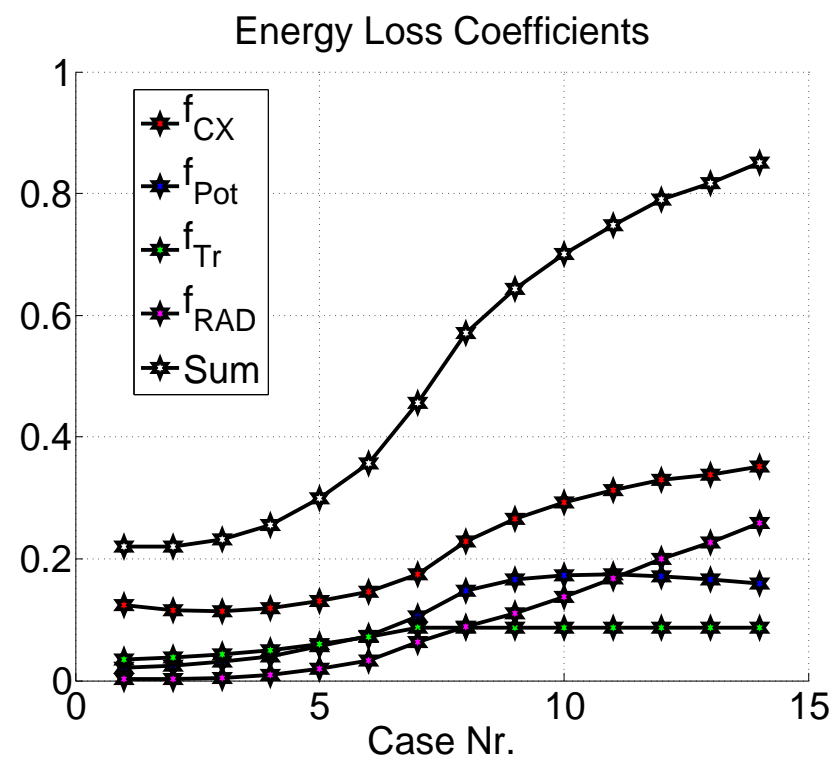

Figure 11: Value of the single fitted energy loss coefficients and of the total lost energy fraction for the cases considered in Fig.10. Note that, in the total loss curve, also the (small) effects of the flux expansion $f_{B}$ are included, its coincidence with the sum of the single coefficients being therefore not exact.

\section{Appendix I: physical assumptions in ASTRA}

In this first appendix, we provide a deeper insight in the physical model employed in ASTRA for the interested readers.

Concerning magnetic equilibrium, plasma elongation $\kappa$ and the triangularity $\delta$ are calculated following [26] and [27], respectively, as

$$
\begin{array}{r}
\kappa=1+0.5 /(A-1) \\
\delta=0.5(\kappa-1) .
\end{array}
$$

The plasma configuration is assumed to be up/down simmetric, i.e. no effects connected to the presence of an X-point are considered (the assumption of single null is however reflected in the fact that only two divertor plates, on which the exhaust power strikes, are considered). Also the safety factor $q$ and the toroidal magnetic field on axis $B_{T}$ are free parameters. Once they are fixed, the total plasma current $I_{p}$ is analytically determined as

$$
I_{p}=4.3 f_{q} \frac{a^{2.0} B_{T}}{(R q)}
$$


with

$$
f_{q}=0.5 \frac{1.17-0.65 / A}{\left(1.0-1 / A^{2}\right)^{2}}\left(1.0+\kappa^{2}\left(1.0+2.0 \delta^{2}-1.2 \delta^{3}\right)\right) .
$$

The corresponding magnetic equilibrium is then calculated inside ASTRA by a coupling with a Grad-Shafranov solver.

Concerning energy transport, the electron temperature at the separatrix $T_{e, s e p}$, in principle, could be determined by means of the $0 \mathrm{D}$ divertor model, see below, but for the sake of simplicity we have decided not to implement such feedback, which would have led to an update of $T_{e, s e p}$ at every timestep. Rather, it has been assumed that $T_{e, s e p}$ remains constant throughout the parameter scan, employing the $0 \mathrm{D}$ model only for the evaluation of $T_{p l}$ and $q_{p l}$. The pedestal top electron temperature $T_{e, P T}$, on the contrary, is determined via a scaling which has been produced by performing 250 runs with the code EPED1 [28] around the DEMO parameters:

$$
T_{e, P T}=2.34 R^{-0.38} \delta^{0.83} I_{p}^{1.25} \kappa^{0.62} \beta_{N}^{0.43},
$$

with $R$ in $\mathrm{m}, I_{p}$ in MA, $\beta_{N}$ being the normalised plasma $\beta$ and $T_{e, P T}$ being expressed in keV. Such scaling yields roughly $T_{e, P T}=5 \mathrm{keV}$ for a typical DEMO 1 scenario, consistently with what was shown in [29]. From pedestal top inwards, the electron temperature profile is calculated assuming a diffusivity $\chi_{e}$ given by

$$
\chi_{e}=1.7 F\left(0.1+3\left(\frac{r}{a}\right)^{4}\right),
$$

with $r$ labelling the radial coordinate and $F$ being an artificial coefficient, whose value is adjusted by ASTRA in the course of the simulation so to fix the global $\mathrm{H}$ factor to the user defined value. For the ions, it is assumed that the temperature at the pedestal top, $T_{i, P T}$, equals $T_{e, P T}$, whereas the corresponding thermal diffusivity $\chi_{i}$ amounts to

$$
\chi_{i}=2 \chi_{e} .
$$

As mentioned in the main text, the particle transport is constrained by fixing the density at the separatrix $n_{u p}$ and the density at the pedestal top $n_{P T}$ at $0.5 n_{G W}$ and $0.9 n_{G W}$ respectively, with $n_{G W}$ indicating the Greenwald density. From pedestal top inwards, the particle transport is governed by a diffusion coefficient $D_{n}$ corresponding to

$$
D_{n}=0.3 \chi_{e}
$$

and an inward pinch velocity $V$ calculated as

$$
V=-2.3 \sqrt{\frac{1}{A}} \frac{D_{n}}{L_{T E}}
$$

with

$$
L_{T E}^{-1}=-\frac{\mathrm{d} \log T_{e}}{\mathrm{~d} r}
$$


No electron source is supposed to be present in the core.

\section{Appendix II: Extension of 0D divertor model}

In this appendix, the main features of the employed 0D SOL and divertor model are shortly presented, insisting in particular on the differences with the previously published version [1]. The interested reader is in any case referred to the mentioned paper for a more complete discussion on the derivation of the equations. The model is based on a set of $1 \mathrm{D}$ equations for the SOL which are then integrated, yielding a system of nonlinear $0 \mathrm{D}$ equations which is very efficient from the computational point of view. The $1 \mathrm{D}$ equations are defined on a SOL open magnetic field line described by a parallel coordinate $x$, with $x=0$ identifying the outer midplane and $x=L_{\|}$identifying the divertor plate:

$$
\begin{array}{r}
q_{\|}(x)=-\chi_{0} f(T) T(x)^{5 / 2} \frac{\mathrm{d} T}{\mathrm{~d} x} \\
\frac{\mathrm{d} q_{\|}}{\mathrm{d} x}=-\sum_{j} c_{z, j} n_{e}(x)^{2} l_{z, j}(T) \\
n_{u p} T_{u p} \Phi(T)=n(x) T(x) \\
q_{\|, p l}=f_{B} \frac{\gamma}{2} e\left(1-f_{m}\right) n_{u p} T_{u p} c_{s 0} \sqrt{T_{p l}} \\
q_{\|, u p}\left(1-f_{C X}-f_{T r}-f_{P o t}-f_{R A D}\right) .
\end{array}
$$

Here, $n(x), T(x)$ and $q_{\|}(x)$ represent the plasma density, temperature and parallel energy flux density profiles along the field line, respectively, with the subscript up indicating the upstream quantities and $p l$ indicating quantities at the plate. Note however that the quantity $q_{\|, p l}$ does not correspond to the heat flux which actually impacts on the divertor plates, but it represents the value of the parallel heat flux at the end of the field line at constant flux-tube cross section. The quantity $\chi_{0} T^{5 / 2}$ (with $\chi_{0}=2390 \mathrm{~W} / \mathrm{m}^{2} / \mathrm{eV}^{7 / 2}$ ) corresponds to the widely employed Spitzer-Härm parallel conductivity, $c_{z, j}$ and $l_{z, j}$ are the relative concentration and the cooling factor of the $j$-th impurity species present in the SOL, $f_{B}=B_{u p} / B_{p l}$ is the flux expansion due to the variation of the strength of the field $B$ (typically around 1 for present tokamaks, but potentially important, for example, for super-X divertor configurations [30]), $\gamma$ is the sheath transmission factor, $e$ is the fundamental charge, $m_{i}$ is the ion mass and $c_{s 0}=\sqrt{2 e / m_{i}}$ is the sound speed corresponding to the temperature of $1 \mathrm{eV}$. Differently from [1], no strong separation between a purely conductive and a purely convective region along the magnetic field line is assumed. Rather, conduction and convection are treated simoultaneously, i.e. a temperature gradient still survives even when conduction is considered. The two functions $f(T)$ and $\Phi(T)$ model the presence of the convective layer, keeping the equations simple from a mathematical point of view. Their derivation can 
be found at the end of this appendix.

The factors $f_{m}, f_{C X}, f_{T r}, f_{P o t}$ and $f_{R A D}$ represent the fraction of momentum and energy losses the plasma undergoes before reaching the divertor plates, their strength ultimately determining the degree of detachment. More in detail,

- $f_{m}$ takes into account the plasma momentum losses,

- $f_{C X}$ takes into account the energy losses due to the neutral hydrogen radiation and the charge exchange reactions (treated simultaneously),

- $f_{T r}$ takes into account the perpendicular transport, which broadens the plasma flow and therefore has to be treated as a net energy loss for a given flux-tube (the same physical phenomenon is often described in the dedicated literature through the parameter $S$, see e.g. $[15,16,31]$ ),

- $f_{P o t}$ takes into account the energy losses due to the ionization of the hydrogen neutrals and

- $f_{R A D}$ takes into account the energy losses due to impurity radiation.

With the only exception of $f_{R A D}$, which is calculated self-consistently using the radiation integral of Eq.40 below (which in turn descends from the integration of Eq.35 and 36), the other factors are calculated iteratively as a function of input and output quantities in the model $\left(n_{u p}, n_{p l}, T_{u p}, T_{p l}, q_{\|, u p}\right.$ and divertor geometry) employing some fitting expressions which have in turn been derived by the analysis of a SOLPS runs database. This approach gives the model a high flexibility (the SOLPS database can be always chosen so to calibrate the fit for a precise machine configuration), together with a quite high degree of precision in calculating the losses, whose correct evaluation is of paramount importance to correctly identify the onset of divertor detachment.

As previously mentioned, the actual 0D model is derived by integrating Eq.35- 
39. The result reads

$$
\begin{array}{r}
q_{\|, u p}^{2}\left(1-f_{R A D}^{2}\right)=2 \chi_{0}\left(n_{u p} T_{u p}\right)^{2}\left[\sum_{j} c_{z, j}^{S O L} \int_{T_{d i v}}^{T_{u p}} \mathrm{~d} T \sqrt{T} f(T) \Phi(T)^{2} l_{z, j}(T)+\right. \\
\left.\sum_{j} c_{z, j}^{d i v} \int_{T_{p l}}^{T_{d i v}} \mathrm{~d} T \sqrt{T} f(T) \Phi(T)^{2} l_{z, j}(T)\right] \\
L_{\|}=\chi_{0} \int_{T_{p l}}^{T_{u p}} \mathrm{~d} T \frac{f(T) T^{5 / 2}}{q_{\|}(T)} \\
L_{d i v}=\chi_{0} \int_{T_{p l}}^{T_{d i v}} \mathrm{~d} T \frac{f(T) T^{5 / 2}}{q_{\|}(T)} \\
q_{\|, p l}=q_{\|, u p}\left(1-f_{C X}-f_{T r}-f_{P o t}-f_{R A D}\right) \\
q_{\|, p l}=f_{B} \frac{\gamma}{2} e\left(1-f_{m}\right) n_{u p} T_{u p} c_{s 0} \sqrt{T_{p l}} .
\end{array}
$$

The unknowns are $T_{u p}, T_{p l}, T_{d i v}$ (which represents the temperature at the divertor entrance), $f_{R A D}$ and $q_{\|, p l}$, whereas $q_{\|, u p}$ and $n_{u p}$ are input. The quantity $L_{d i v}$ indicates the connection length at the divertor entrance, and the impurity concentrations have been split between SOL $\left(c_{z, j}^{S O L}\right)$ and divertor region $\left(c_{z, j}^{d i v}\right)$, neglecting otherwise their spatial dependence. The function $q_{\|}(T)$ can straightforwardly be derived from Eq.40 performing the integration between $T_{p l}$ and the arbitrary temperature $T$ instead of $T_{u p}$. The actual heat flux reaching the target plate $q_{p l}$ is calculated a posteriori as

$$
q_{p l}=\frac{q_{\|, p l}}{\eta_{B} f_{B}}+\frac{e \Gamma_{\|, p l}}{\eta_{B}} E_{R e c}
$$

where $E_{R e c}=20 \mathrm{eV}$ is the energy associated to surface recombination, $\eta_{B}$ is the geometrical factor which takes into account the inclination of the magnetic field line with respect to the target plate (its value is around 10 for DEMO [5]) and

$$
\Gamma_{\|, p l}=\frac{q_{\|, p l}}{\gamma e T_{p l} f_{B}} .
$$

is the parallel particle flux at the plate.

The SOLPS database which has been employed for the calibration of the loss factor fits contains about 40 ASDEX Upgrade and DEMO related runs, with conventional and closed divertor configuration. The agreement of the model with the SOLPS runs within the database turns out to be quite satisfactory, as shown in Fig.10, especially at low temperature and high losses, which is the most relevant limit in a reactor framework, as a detached divertor is required. In particular, we stress that the so called "roll-over", i.e. the decrease of parallel particle flux at lower temperatures which identifies the onset of detachment, is correctly reproduced by our model. Fig. 11 shows the value for the fitted loss 
parameters corresponding to the cases in Fig.10, clearly indicating that even at relatively high temperature at the plate, their role is absolutely non negligible.

Finally, the functions $f(T)$ and $\Phi(T)$ are introduced to take into account the potential presence of convective transport along the SOL open magnetic field line. Their role is to smoothly match the region of pure conduction with the conductive/convective one, leaving the problem treatable from a mathematical point of view. When heat convection is negligible, the heat transport is sustained by conduction only. Employing the sheath boundary condition Eq.38, the constraint

$$
f_{B} \frac{\gamma}{2} e\left(1-f_{m}\right) n_{u p} T_{u p} c_{s 0} \sqrt{T_{p l}}=-\left.\chi_{0} T_{p l}^{5 / 2} \frac{\mathrm{d} T}{\mathrm{~d} x}\right|_{x=L_{\|}}
$$

must be fulfilled at the plates. On the contrary, if also conduction is present before the sheath entrance, Eq.47 reads

$$
\begin{array}{r}
f_{B} \frac{\gamma}{2} e\left(1-f_{m}\right) n_{u p} T_{u p} c_{s 0} \sqrt{T_{p l}}=-\left.\chi_{0} T_{p l}^{5 / 2} \frac{\mathrm{d} T}{\mathrm{~d} x}\right|_{x=L_{\|}}+ \\
3 f_{B} e\left(1-f_{m}\right) n_{u p} T_{u p} c_{s 0} \sqrt{T_{p l}}
\end{array}
$$

having employed a standard Braginskij expression for the convective term, with Mach number $M=1$. With straightforward algebra, Eq.48 can be re-written as

$$
f_{B} \frac{\gamma}{2} e\left(1-f_{m}\right) n_{u p} T_{u p} c_{s 0} \sqrt{T_{p l}}=-\left.\chi_{0}\left(\frac{\gamma}{\gamma-6}\right) T_{p l}^{5 / 2} \frac{\mathrm{d} T}{\mathrm{~d} x}\right|_{x=L_{\|}}
$$

It is assumed that the convection term becomes non-negligible at a certain temperature, labelled $T_{c}$, below which recycling neutrals can sufficiently penetrate in the plasma before undergoing a ionization event, thus returning to the divertor plate having been accelerated up to a sufficiently high speed $(M \sim 1$, as previously stated). Following [32], we set this critical temperature at $15 \mathrm{eV}$. Eq.47 and 48 can be thus summarised in a single one introducing the auxiliary function $f(T)$ as

$$
f_{B} \frac{\gamma}{2} e\left(1-f_{m}\right) n_{u p} T_{u p} c_{s 0} \sqrt{T_{s h}}=-\chi_{0} f(T) T_{p l}^{5 / 2} \frac{\mathrm{d} T}{\mathrm{~d} x},
$$

where $f(T) \rightarrow 1$ for $T>T_{c}$ and $f(T) \rightarrow\left(\frac{\gamma}{\gamma-6}\right)$ for $T<T_{c}$. To have a smooth but quick transition, we set $f(T)$ as an hyperbolic tangent:

$$
\begin{array}{r}
\alpha=\left(\frac{\gamma}{\gamma-6}\right) \\
f(T)=\frac{\alpha-1}{2} \tanh \left(T_{c}-T\right)+\frac{\alpha+1}{2}
\end{array}
$$


The constraint on the total pressure conservation has been treated on the same footing. If only conduction were present, the total pressure corresponds everywhere to the static pressure, namely

$$
n_{u p} T_{u p}=n(x) T(x)
$$

On the contrary, where convection is present (having assumed $M=1$ )

$$
\frac{n_{u p} T_{u p}}{2}=n(x) T(x)
$$

As before, we summarise the two limits as

$$
n_{u p} T_{u p} \Phi(T)=n(x) T(x)
$$

where $\Phi(T) \rightarrow 1$ for $T>T_{c}$ and $\Phi(T) \rightarrow \frac{1}{2}$ for $T<T_{c}$. A possible choice is

$$
\Phi(T)=\frac{3}{4}-\frac{1}{4} \tanh \left(T_{c}-T\right) .
$$

The present approach differs, for example, from the one presented in [24], where all relevant quantities are determined with fitting laws, as in our case the model mostly relies on first-principle based equations.

\section{References}

[1] M. Siccinio et al., Plasma Phys. Control. Fusion 58, 125011 (2016)

[2] G. V. Pereverzev, IPP Report 5/42 (1991)

[3] E. Fable et al., Plasma Phys. Control. Fusion 55, 124028 (2013)

[4] H. Zohm et al., Nucl. Fusion 53, 073019 (2013)

[5] R. Wenninger et al., Nucl. Fusion 57, 046002 (2017)

[6] F. Romanelli et al., A roadmap to the realisation of fusion energy, EFDA Document (2012)

[7] M. Kovari et al., Fusion Eng. and Des. 89, 3054 (2014)

[8] M. Kovari et al., Fusion Eng. and Des. 104, 9 (2016)

[9] ITER Physics Expert Groups on Confinement and Transport and Confinement Modelling and Database, ITER Physics Basis Editors, Chapter 2: Plasma confinement and transport, Nucl. Fusion 39, 2175 (1999).

[10] N. A. Uckan, ITER Physics Design Guidelines, ITER-TN-PH-8-6 (1989)

[11] G. Giruzzi et al., Nucl. Fusion 55, 073002 (2015) 
[12] H. Zohm et al., Nucl. Fusion 57, 086002 (2017)

[13] R. Wenninger et al., Nucl. Fusion 54, 114003 (2014)

[14] G. Federici et al., Nucl. Fusion 57, 092002 (2017)

[15] T. Eich et al., Phys. Rev. Lett. 107, 215001 (2011)

[16] T. Eich et al., Nucl. Fusion 53, 093031 (2013)

[17] A. E. Costley, J. Hugill and P. F. Buxton, Nucl. Fusion 55, 033001 (2015)

[18] F. Albajar, J. Johner and G. Granata, Nucl. Fusion 41, 665 (2001)

[19] Y. R. Martin et al., J. Phys.: Conf. Ser. 123, 012033 (2008)

[20] R. Wenninger et al., Nucl. Fusion 57, 016011 (2017)

[21] M. L. Reinke, Nucl. Fusion 57, 034004 (2017)

[22] M. S. Chu and M. Okabayashi, Plasma Phys. Control. Fusion 52, 123001 (2010)

[23] G. H. Neilson (edited by), Magnetic fusion energy from experiments to power plants, Woodhead Publishing, ISBN 978-0-08-100326-8

[24] G. W. Pacher et al., Nucl. Fusion 47, 469 (2007)

[25] A. G. Peeters et al., Nucl. Fusion 47, 1341 (2007)

[26] H. Zohm et al., Nucl. Fusion 53, 073019 (2017)

[27] W. Biel et al, accepted by Fusion Eng. and Des.

[28] P. B. Snyder et al., Phys. Plasmas 16, 056118 (2009)

[29] R. Wenninger et al., Nucl. Fusion 55, 063003 (2015)

[30] P. M. Valanju et al., Phys. Plasmas 16, 056110 (2009)

[31] A. Scarabosio et al., Journ. Nucl. Mat. 463, 49 (2015)

[32] A. Leonard et al., Nucl. Fusion 52, 063015 (2012) 